\title{
Selección de áreas prioritarias para la conservación de flora gipsícola en el sureste de la Península Ibérica
}

\author{
Selection of priority areas for the conservation of gypsophilous flora in southeast \\ Iberian Peninsula
}

\author{
MANUEL I. CERRILLO, ELÍAS D. DANA, HERMELINDO CASTRO, M. LUISA RODRÍGUEZ-TAMAYO \\ \& JUAN F. MOTA
}

\author{
Departamento de Biología Vegetal y Ecología, Facultad de Ciencias Experimentales, \\ Universidad de Almería, E-04120, Almería, España; \\ e-mail: cerrillo@ual.es; jmota@ual.es
}

\begin{abstract}
RESUMEN
Estudios recientes han demostrado que los afloramientos de yeso de la provincia de Almería (sureste de España) son los de mayor valor desde el punto de vista de la conservación de la flora gipsícola ibérica. La necesidad de conservar este patrimonio unida a la imposibilidad real de proteger todos los afloramientos existentes en la región, nos llevó a elucidar qué áreas de la provincia deberían ser consideradas como prioritarias para lograr la conservación de la flora gipsícola de estos enclaves. Para ello, se estableció como objetivo prioritario lograr la representación de todas las especies al menos una vez, a la vez que se plantearon otros escenarios posibles en los que se podría basar la elección de áreas -objetivos complementarios-. Se elaboró la cartografía de los gipsófitos, referida a celdas de 10 km de lado y se evaluó la importancia de cada enclave según diferentes criterios utilizados comúnmente en los estudios sobre selección de áreas (diversidad de especies, rareza de la flora, complementariedad) contrastando los resultados obtenidos mediante cada uno de ellos. Los resultados mostraron que la protección de los afloramientos existentes en tan sólo tres cuadrículas (Sorbas, ya protegida principalmente por sus valores geomorfológicos, Venta de los Yesos y Topares) sería suficiente para lograr la representación de todos los gipsófitos de la región y alcanzar además varios de los objetivos complementarios de conservación. Los resultados se discuten teniendo en cuenta las peculiaridades sociales y administrativas del territorio.
\end{abstract}

Palabras clave: biodiversidad, afloramientos de yeso, áreas prioritarias, criterios de conservación, microreservas.

\begin{abstract}
Recent studies have highlighted the gypsum outcrops of Almería (southeast Spain) as the most outstanding for the conservation of Iberian gypsophilous flora. The need of preserving this natural heritage and the impossibility to protect all the outcrops present within the territory, led us to identify which areas should be considered as of priority for conservation. To this end, we establish as a priority that the proposed conservation network of sites should include all gypsophyte species at least once. Other possible conservation goals on which to base the area selection and the associated scenarios were also taken into account. We first elaborated a cartography for the gypsophytes based on 10 $\mathrm{km}$ sided-cells. Next, in order to rank the importance of each locality, several commonly employed conservation criteria (diversity, rarity of the flora, complementarity) were applied to the cartographic information, and the findings obtained by each procedure contrasted. The results showed that protecting the deposits found in three cells (Sorbas, already protected because of its geomorphological singularity, Venta de los Yesos and Topares) would allow the representation of each species at least once and to achieve several complementary conservation targets. The findings are discussed within the context of the social and administrative peculiarities of the area.
\end{abstract}

Key words: biodiversity, gypsum outcrops, areas selection, conservation criteria, microreserves.

\section{INTRODUCCIÓN}

La Península Ibérica es uno de los territorios con mayor valor para la conservación de la flora europea, ya que cuenta con un elenco florístico que resalta tanto por su riqueza en especies, por el número de endemismos ibéricos (Simón 1994) y el número de especies amenazadas que concentra
(Domínguez-Lozano 2000). La singularidad de la flora ibérica se debe a múltiples factores (NietoFeliner 1999) entre los que se encuentran los de tipo paleoclimático (Crisis mesiniense, glaciaciones pleistocenas), geográficos, ecológicos (ecotonos, refugios, variedad de hábitats) y la superposición de los efectos generados por ellos (Montserrat \& Villar 1972, Gómez-Campo \& Malato-Beliz 1985). 
En los ambientes de insularidad edáfica como los afloramientos de yeso -o "aljezares", del término árabe "aljez", mineral de yeso- la originalidad de la flora ibérica se acentúa. En los aproximadamente $35.487 \mathrm{~km}^{2}$ que, según Riba \& Macau $(1962)^{1}$ ocupan estos sustratos en la Península (i.e., cerca del $12 \%$ del territorio español), se encuentran al menos 38 taxa cuya presencia está ligada a estos substratos restrictivos (Dana 2000), todos ellos endémicos o raros en el contexto del Arco Mediterráneo (Braun-Blanquet \& Bolòs 1957, Rivas-Goday 1957, Mota et al. 1993, Loidi \& Costa 1997). Además, al menos 10 especies vasculares ligadas a este tipo de suelos -i.e., cerca de un $25 \%$ de las especies gipsófilas- se encuentran bajo algún grado de amenaza (Dana 2000, Domínguez-Lozano 2000).

La singularidad de los aljezares ibéricos ha sido reconocida también internacionalmente, ya que sus comunidades vegetales y algunas de las especies que las conforman están catalogadas como prioritarias por la Directiva Hábitats (Anon 1992), un documento firmado por varios países miembros de la Unión Europea enfocado hacia la preservación, a escala europea, de las especies vegetales, animales y fitocenosis de mayor valor biológico. Si bien este acuerdo no obliga -aunque recomienda- a cada estado firmante a la preservación directa de todas las especies y comunidades que en él se recogen, no carece de valor, ya que constituye la base para priorizar áreas de conservación dentro de la "Red Natura 2000" (Ostermann 1998), o lo que es lo mismo, el entramado de espacios naturales protegidos de la Comunidad Europea.

Pese a este reconocimiento internacional, en España no existen afloramientos de yesos protegidos específicamente por sus valores botánicos, aunque algunos depósitos quedan casualmente incluidos dentro de algún espacio protegido, seleccionado por otro tipo de valores (faunísticos, geomorfológicos, paisajísticos, etc.). Por tanto, resulta evidente la necesidad de que, al menos los afloramientos más valiosos para la conservación de la flora gipsícola, cuenten con algún grado de protección legal. Debido a que los depósitos más valiosos para la conservación se localizan en el sureste ibérico -especialmente en la provincia de Almería-, cualquier planteamiento de protección de la flora de yeso pasa por la conservación de, al menos, una parte de los aljezares de esta región (Mota et al. 1993, Dana 2000).

${ }^{1}$ RIBA O \& F MACAU (1962) Situación, características y extensión de los terrenos yesíferos en España. Primer Coloquio Internacional sobre las obras publicadas en los terrenos yesíferos, Sevilla-Madrid-Zaragoza, España: 5-33.
Sin embargo, antes de seleccionar áreas prioritarias para su integración en redes de espacios protegidos, es imprescindible definir claramente cuál es el objetivo de conservación que se persigue. A este respecto, la mayoría de los trabajos tratan de lograr la protección de la mayor cantidad de especies posibles o de asegurar la representación de todas las especies raras, endémicas o amenazadas, generalmente en el mínimo espacio posible debido a los costes asociados a la conservación de la biodiversidad (Margules \& Usher 1981, Kirkpatrick 1983, Margules et al. 1988, Myers 1990, Margules \& Austin 1991, Castro-Parga et al. 1996, Hernández \& Bárcenas 1996, Moreno-Saiz et al. 1996, 1998). La propuesta final de áreas a proteger surge de la aplicación simultánea de varios criterios y de la confrontación de los resultados obtenidos según cada uno de ellos (e.g., Dinerstein \& Wikramanayake 1993, Pressey et al. 1993, Williams et al. 1996a).

De acuerdo con estos antecedentes, en este trabajo se fija como objetivo prioritario de conservación que todas las especies gipsícolas del área queden incluidas en al menos una de las localidades y que ello se logre protegiendo el mínimo número de enclaves posible. Simultáneamente se analizan y discuten varias posibilidades de selección de áreas prioritarias según se pretenda lograr alguno de los siguientes objetivos complementarios: (1) proteger las áreas con mayor riqueza de especies, considerando tanto la riqueza total como la riqueza en especies endémicas y amenazadas, (2) proteger todas las localidades que cuenten elementos endémicos, y (3) proteger todos los enclaves que alberguen especies bajo algún grado de amenaza.

En cualquier análisis de conservación debe considerarse también el grado de amenaza real existente sobre el elemento objeto del análisis (especies, comunidades, localidades prioritarias) y estudiar otros factores que puedan influir en la viabilidad de las propuestas (Williams et al. 1996a, 1996b). Por ello, se analiza también el grado y fuentes de amenaza de los aljezares estudiados y la posible adecuación de las diferentes medidas y figuras legales de protección, teniendo en cuenta, a su vez, la realidad administrativa y social del territorio. Se espera además que este trabajo sea una herramienta útil en la que las autoridades implicadas en la conservación puedan basar la toma de decisiones sobre la selección de aljezares a proteger. 


\section{MATERIALES Y MÉTODOS}

Área de estudio: características del medio biofísico

El territorio estudiado corresponde a la provincia de Almería (sureste de España, $3^{\circ} 9^{\prime} \mathrm{O}, 37^{\circ} 55^{\prime} \mathrm{N}$ ), que cuenta con una extensión de $8.774 \mathrm{~km}^{2}$ (Fig. 1). El $23,8 \%\left(2.088 \mathrm{~km}^{2}\right)$ de esta superficie corresponde a afloramientos de yeso, generados esencialmente durante el Mioceno, con una cristalización masiva y estando casi la totalidad de ellos sometidos actualmente a un clima semiárido, con precipitaciones por debajo de los $300 \mathrm{~mm}$ anuales. Los depósitos se encuentran bastante fragmentados, apareciendo generalmente como reducidos afloramientos con una extensión que en su mayoría oscila entre 0,25 y $14 \mathrm{~km}^{2}$ (Lázaro 1984).

Aunque existen otros afloramientos en la Península Ibérica, se ha escogido la provincia de Almería ya que sus afloramientos son los más valiosos de cara a la conservación de la flora y comunidades gipsícolas ibéricas, tanto del componente vascular (Mota et al. 1993, Dana 2000) como criptogámico (Gutiérrez 1990, Gutiérrez \& Casares 1994, Guerra et al. 1995, Egea \& Alonso 1997). La riqueza total de especies y la abundancia de elementos raros, endémicos y amenazados que presenta esta región la convierten en un verdadero punto caliente -en el sentido de Myers (1988, 1990) y Myers et al. (2000)- para la conservación de esta clase de especies. En efecto, a pesar de que los terrenos yesíferos de esta región sólo representan el 5,9\% del total nacional, concentran el $51 \%$ de las especies gipsófilas del país, de las que seis son endémicas de la provincia de Almería. La región alberga además seis de los al menos diez taxa asociados a yesos que actualmente están amenazados en España (Dana 2000).

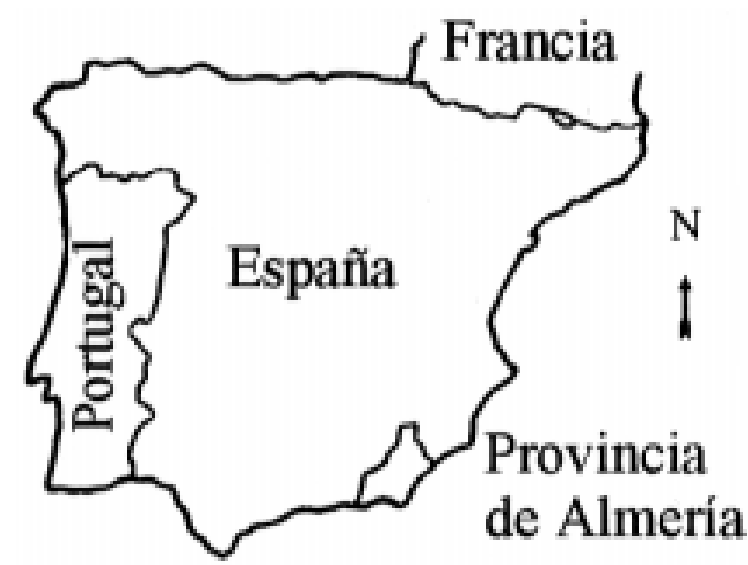

Fig. 1: Localización geográfica de la zona de estudio.

Geographical location of the studied outcrops.

\section{Características administrativas y sociales}

Es necesario indicar que la provincia de Almería pertenece administrativamente a la Comunidad Autónoma de Andalucía, que posee la mayor parte de las competencias en materia medioambiental. Precisamente todos los afloramientos de Sorbas y algunos de las localidades de Los Castaños y Río Aguas están protegidos mediante la figura de "Paraje Natural" -Paraje Natural del Karst en Yesos de Sorbas-, específica de la legislación ambiental andaluza. Sin embargo, la selección de localidades para su declaración como espacio protegido no tiene por qué coincidir con las que resultarían prioritarias desde el punto de vista de la biodiversidad vegetal, ya que su declaración se fundamentó principalmente en sus valores geomorfológicos (Mota et al. 1997). En efecto, dentro del área protegida existe una de las formaciones kársticas en yeso más extensas y peculiares del mundo (Calaforra \& Forti 1991, Calaforra \& Pulido 1997, Calaforra 1998). Dentro de este entorno protegido, se prohíbe la práctica de la minería, lo que indirectamente ha restringido la destrucción de la flora gipsícola de estos enclaves. Sin embargo se permiten otras prácticas como la agricultura, que también conllevan consecuencias negativas para la flora de yesos (Dana 2000).

Actualmente los aljezares almerienses se encuentran francamente amenazados por la extracción del mineral de yeso, ya que Almería es la mayor productora de yeso de España. Esta amenaza llega a los límites del Paraje Natural, donde también se localizan explotaciones a cielo abierto. Hay que resaltar que la minería constituye la base de la economía de la comarca de Sorbas, que cuenta con una población de unos tres mil habitantes. Esta circunstancia contrasta con el impacto ecológico que genera la actividad minera, que ya ha provocado la desaparición de numerosas poblaciones de gipsófitos en las zonas no protegidas de la comarca (Cerrillo et al. 2001a). De este modo, los condicionantes sociales y administrativos que concurren en la zona de estudio, junto a los requerimientos ecológicos de las especies de suelos yesíferos pueden determinar en gran medida qué formas de protección de las áreas seleccionadas resultarían más viables.

\section{Elucidación de áreas prioritarias}

Para la elucidación de áreas prioritarias, y de acuerdo con la terminología y clasificación propuesta por Rivas-Martínez \& Costa (1970) y Escudero et al. (1997) se emplearon los 19 
"gipsófitos" (i.e., cuya presencia está exclusivamente ligada a los yesos) y "gipsóvagos preferentes" (i.e., aquellas especies que, si bien puntualmente pueden aparecer en otros tipos de substratos, presentan una marcada preferencia por los depósitos yesíferos) que habitan los afloramientos de la provincia de Almería. Por comodidad, emplearemos frecuentemente el término "gipsófito" en su sentido amplio, incluyendo también a los "gipsóvagos preferenciales".

Estas 19 especies son las que presentan mayor valor de conservación y las que distinguen florísticamente los afloramientos de la provincia. A diferencia de otros elementos florísticos, su conservación solo puede llevarse a cabo protegiendo los aljezares. Los distintos grupos de afloramientos de la provincia de Almería se distinguen entre sí esencialmente por las diferencias en la composición de la flora gipsófila que sustentan y no por la presencia o ausencia de las especies no ligadas a los yesos, comunes a todos los afloramientos (ver Lázaro 1984, 1986, Mota et al. 1998) y a otros territorios no gípsicos. Con esta metodología se puede asegurar que la selección de los afloramientos se basará sólo en las especies que los caracterizan y que les confieren su singularidad florística, y no por otras cuya ecología no está ligada a los yesos y cuya protección puede plantearse en una amplia gama de ambientes y territorios circundantes.

De cada uno de los 19 gipsófitos empleados en el análisis se recopiló toda la información relativa a su distribución en el territorio de estudio (Lázaro 1984, 1986, Sagredo 1987, Sanz \& Costa 1987, Cueto 1989, Molina et al. 1989, Rubio-Sánchez 1990, Rubio-Sánchez \& Escudero 1992, RubioSánchez et al. 1992, Mota et al. 1997, 1998, Cerri11 o et al. 2001b), situándolos en las cuadrículas geográficas UTM de $10 \mathrm{~km}$ de lado correspondientes al huso 30S. La información bibliográfica se completó y contrastó con diversas salidas de campo a los afloramientos de la región, elaborando finalmente una matriz de presencia y ausencia de especies en las cuadrículas. De acuerdo con la metodología propuesta por Williams (1994, 1999), Williams et al. (1996a), Humphries et al. (1995) y Castro-Parga et al. (1996), para identificar los aljezares prioritarios para la conservación se han empleado tres criterios básicos: riqueza, rareza y complementariedad. El modo en que fueron aplicados se describe a continuación.

\section{Riqueza de especies (Rn)}

Se calculó el número de especies -riqueza- en cada cuadrícula (Haila \& Kouki 1994). Además, de forma semejante al procedimiento aplicado por diversos autores (e.g. Hernández \& Bárcenas 1996), se calculó tanto la riqueza de endemismos regionales -entendiendo como tales las especies restringidas exclusivamente a la provincia de Almería- como la riqueza de especies amenazadas en cada enclave. En este último caso se consideraron las especies que actualmente se encuentran bajo alguna de las categorías de amenaza de la UICN según Domínguez-Lozano (2000).

Siguiendo el criterio de Prendergast et al. (1993) se ha escogido el cinco por ciento de las cuadrículas con mayor valor de riqueza -en cualquiera de sus posibilidades- como las de mayor concentración de especies y que por tanto deberían ser consideradas en la selección de áreas.

\section{Rareza continua $(R c)$}

Siguiendo la metodología estándar para el cálculo de la rareza (Usher 1986, Howard 1991, Williams 1993) se calcularon los valores de rareza para cada especie mediante la expresión $r_{i}=Q^{-1}$, siendo $\mathrm{Q}$ el número total de cuadrículas en donde aparece la especie i. De este modo, cuanto menor sea el número de cuadrículas en las que aparece una especie dada, mayor será su rareza. Según Kerr (1997) se puede estimar también la importancia que una cuadrícula presenta ("rareza continua") según la cantidad de especies raras que concentra. Cuanto mayor sea el número de especies raras, i.e., se localizan en una o unas pocas celdas, que aparecen en una localidad, mayor será su rareza. Para una localidad dada, su valor de rareza continua vendría dado por la expresión Rc $=\sum r_{i}$.

\section{Rareza discontinua $(R d)$}

Siguiendo la recomendación de Gaston (1997) se aplicó también otra medida derivada del concepto de rareza continua, la de rareza discontinua $(\mathrm{Rd})$, que se calcula de manera análoga a la Rc, pero considerando sólo un porcentaje (del 20-30\% según Gaston 1997) de las especies más raras. En adelante a estas especies se les denominará como "muy raras". Este porcentaje se calcula ordenando las especies en orden decreciente de valores de $\mathrm{r}_{\mathrm{i}}$, eligiendo después el 20-30\% de las más raras. Debido a la existencia de empates en los valores de $r_{i}$, para calcular la $\mathrm{Rd}$ de cada localidad se utilizó el $26 \%$ de las especies (cinco especies) de los 19 gipsófitos existentes en el área: Campanula fastigiata, Rosmarinus $x$ lavandulaceus, Frankenia thymifolia, Teucrium balthazaris y Jurinea pinnata. 


\section{Complementariedad}

Este concepto tiene en cuenta la magnitud en que la adición de una nueva localidad incrementa el número de especies recogidas en una red de reservas (Vane-Wright et al. 1991, Colwell \& Coddington 1994). En este estudio se aplicó este concepto tanto a los valores de riqueza total de las cuadrículas como a los de riqueza de endemismos, riqueza de especies amenazadas y riqueza de especies muy raras.

\section{RESULTADOS Y DISCUSIÓN}

Valor de las localidades para la conservación: riqueza total de especies

La cuadrícula de Sorbas (WG70) alberga la mayor concentración de gipsófitos (contiene el $68 \%$ de los gipsófitos de Almería, i.e., 13 especies), aunque está seguida de cerca por las cuadrículas correspondientes a la Venta de los Yesos (WG60), Río Aguas (WG80) y Los Castaños (WG81) (Tabla 1 y Fig. 2). Cada una de estas tres últimas celdas contienen el $63 \%$ de los taxa gipsófitos del territorio (12 especies). Por tanto, según el criterio de Prendergast et al. (1993) estas cuadrículas (5\%) deberían ser consideradas como prioritarias
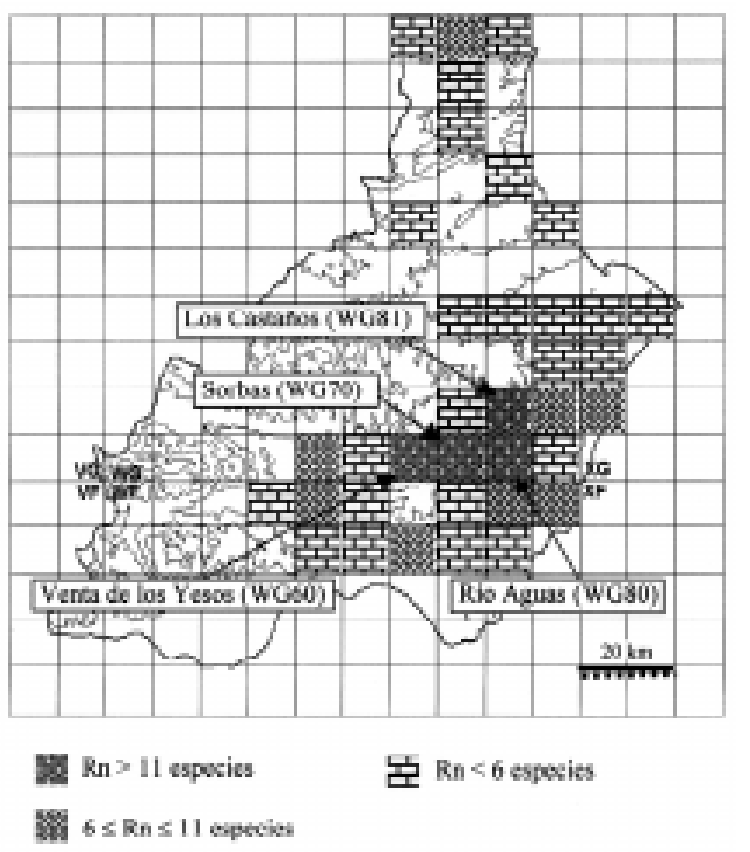

Fig. 2: Intervalos de riqueza en cada cuadrícula. Richness intervals in each cell. para preservar áreas de elevada diversidad. Hay que destacar que la cuadrícula WG91 de Turre también posee una riqueza relativamente elevada (10 taxa), al igual que las localidades de Mojácar (XG01) y Carboneras (WF89), ambas con nueve especies y la Loma de los Yesares (WF99) y Base de Sierra Alhamilla (WF49) con ocho especies cada una.

Valor de las localidades para la conservación: riqueza de especies endémicas

Cada una de las siguientes cuadrículas, ordenadas en sentido decreciente según su riqueza total, alberga a cinco de las seis especies endémicas del territorio: WG70 (Sorbas), WG80 (Río Aguas), WG81 (Los Castaños), WG91 (Turre), WF89 (Carboneras), XG01 (Mojácar) y WF99 (Loma de los Yesares) (Fig. 3A). Por ello, la protección de cualquiera de estos enclaves permitiría que el 83 $\%$ los taxa endémicos quedasen representados al menos una vez. La celda de Venta de los Yesos (WG60), a pesar de contar con una elevada riqueza específica (12 especies), recoge sólo dos especies endémicas de Almería. Otras cuadrículas, e.g., WG79 (Topares), no presentan ningún elemento exclusivo de la región. Como se observa en la Fig. 3A, la protección de todas las poblaciones de endemismos implicaría a un elevado número de localidades.

Valor de las localidades para la conservación: riqueza de especies amenazadas

La localidad con mayor número de especies amenazadas fue de nuevo Sorbas (WG70), con seis especies (Fig. 3B). Cinco especies amenazadas se encuentran en cada una de las cuadrículas WG80, WG81 (ambas con 12 gipsófitos) y WF89 (con nueve especies en total). Debido a este empate entre los tres últimos enclaves las cuatro celdas deberían ser consideradas prioritarias si se persiguiera preservar al menos el cinco por ciento de las localidades con mayor concentración en especies amenazadas. Es interesante destacar que las localidades de Turre (con 10 gipsófitos), Loma de los Yesares (ocho especies), y Mojacar (que posee nueve especies) recogen cuatro taxa amenazados. Por el contrario Venta de los Yesos y WF58 contienen sólo dos de las seis especies amenazadas de la región. Como se observa en la Fig. 3B, la protección de todos los puntos donde aparecen poblaciones de las especies amenazadas implicaría nuevamente a un elevado número de enclaves. 


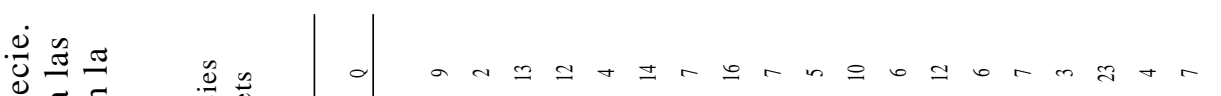

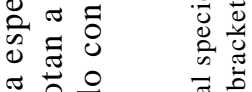

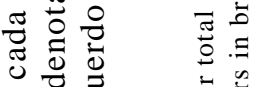

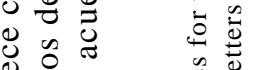

¿

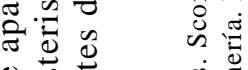

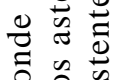

这

¿

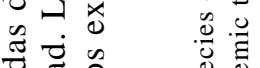

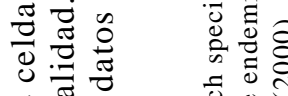

ช

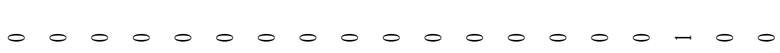

- $\stackrel{0}{\dot{0}} 0$

$00000-0-0000-000-1-0$

in $\overrightarrow{5} 0$

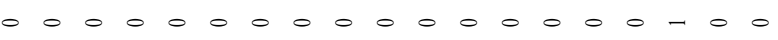

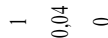

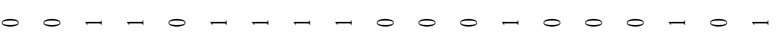

a ${ }_{0}^{\infty} 0$

$000000000-00000000$

$-\overrightarrow{0} 0$

$00000-0-0000-000-1-0$

in $\vec{n} 0$

$00000-0-0000-000-1-0$

in $\vec{z} 0$

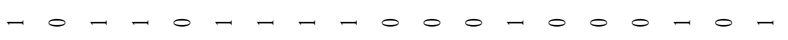

$\therefore \stackrel{2}{\circ} 0$

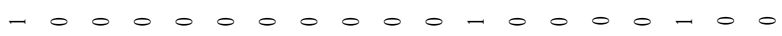

ก กิ

$000000000000-000000$

$-\stackrel{\infty}{0} 0$

บ

$00000000-000000-00$

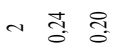

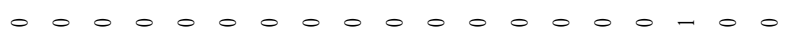

-

$-0--0--1-00-1-00-0-$

$\simeq$ בิ 0

ב

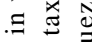

๘

$\stackrel{0}{0} \stackrel{0}{0}$

응

$-0-10---1,00--1,00-0-$

$\simeq$ בे 0

- $0000-0-0-1-0-0000-0$

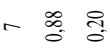

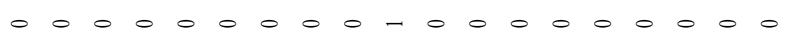

- ํㅡㅇ ำ

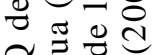

ป

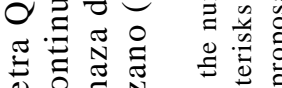

至证

感艺

(

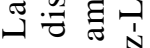

文

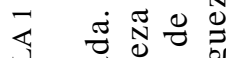

0 응

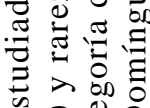

ذ) Ð

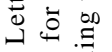

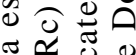

ชं

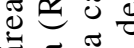

ส

ส

ఫ

芯司哭

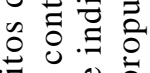

击

‡:

宓䒕苞

$000000000-000000000$

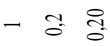

$000000000000000-00$

-

$0000000000-0000000$

$-\cong 0$

$0---0---$ - $-00--1-0-0-$

$=\stackrel{\infty}{-}:$

$0000000000-00000000$

- $\overrightarrow{0} 0$

$000000000-000000000$

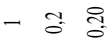

$0----$ - $-0,00-0----1-0$

$\simeq \stackrel{\vec{\sigma}}{-} \stackrel{0}{-}$

$00000-0-00-000-0-00$

in :

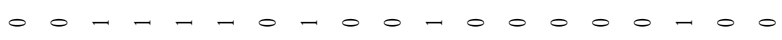

-

$-0-100-0-0000-00-0-$

$\infty$ हे 0

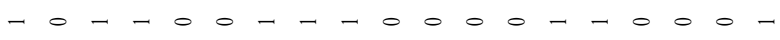

$a \stackrel{5}{5} 0$

$00-00000000-0000000$

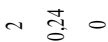

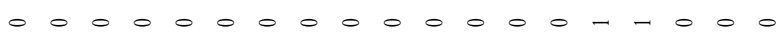

$\sim \stackrel{\infty}{0}$ ?

$000-000000000000-00$

$\sim \stackrel{m}{0} 0$

至苛

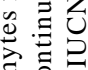

to

$00---10-00-00000-00$

-

- $000000-00-000-1-000$

in $\cong$

$00-00000000000-0000$

$\sim$ ปิ

$-0----_{-}-0,00000-00$

$\infty \stackrel{\circ}{\infty} \stackrel{0}{0}$

$0000000000-00000-00$

$\sim \stackrel{\overrightarrow{0}}{\overrightarrow{0}} 0$

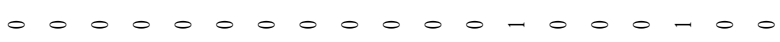

$\sim \stackrel{2}{0} 0$

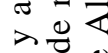

$\exists$ 定

훙

중 


\section{Rareza continua}

La cuadrícula WG60 de Venta de los Yesos alcanzó el mayor valor de rareza, con $\mathrm{Rc}=1,9$, seguida por Sorbas (WG70) con Rc = 1,82 (Fig. 4A). Las celdas WG80 y WG81, contiguas a la anterior, también presentaron valores de rareza continua relativamente elevados $(\mathrm{Rc}=1,29$ en ambos casos), seguidas por WF89, WG81 y WF99 con valores próximos a la unidad.

\section{Rareza discontinua}

Con este parámetro, la rareza de la flora de Venta de los Yesos se hizo más notable aún, ya que las diferencias entre los valores de las cuadrículas se acentuaron considerablemente. Así, Venta de los Yesos obtuvo un $\mathrm{Rd}=1,08$, mientras que Sorbas sólo alcanzó un valor de 0,50 , lo que significa que la celda WG60 (Venta de los Yesos) alberga la mayor concentración de especies muy raras (Fig.
$\boldsymbol{A}$

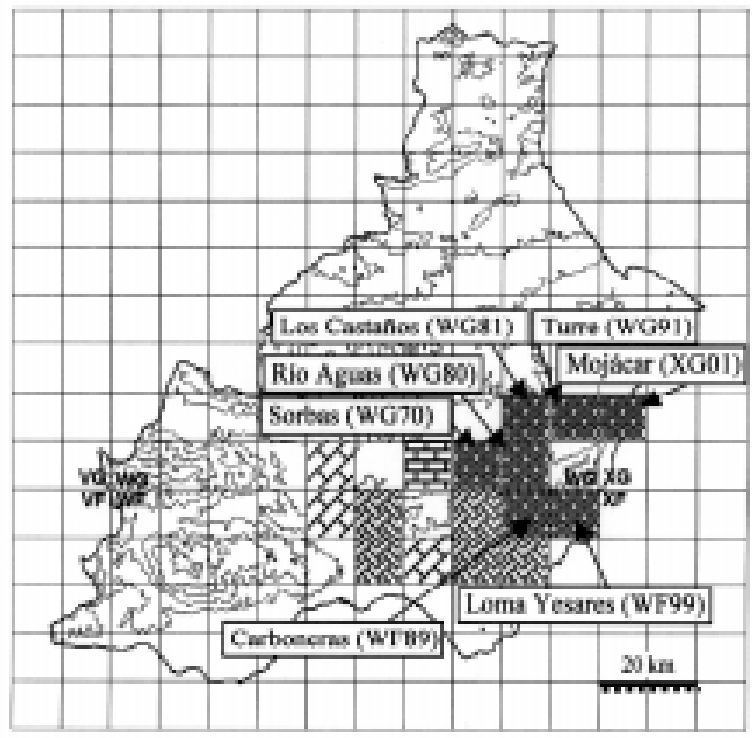

B

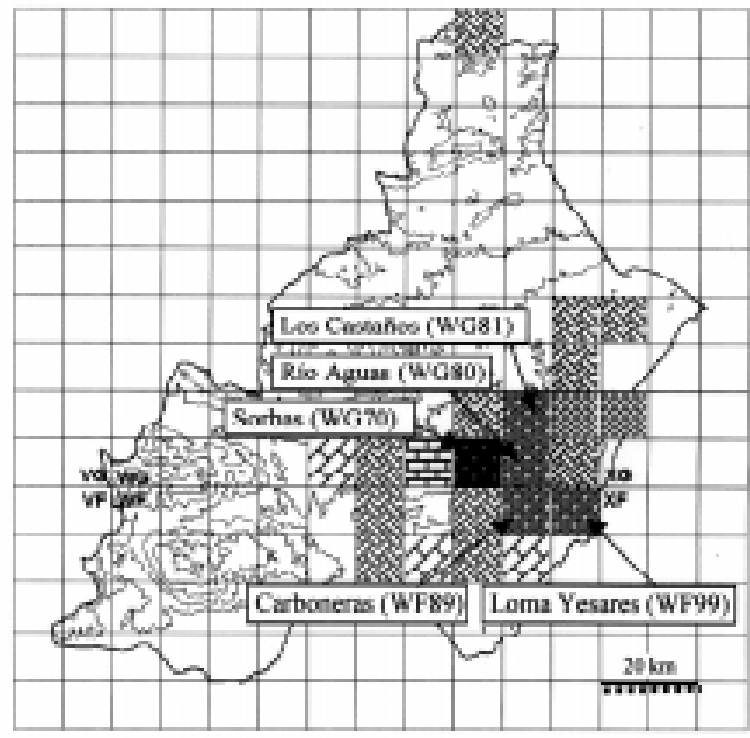

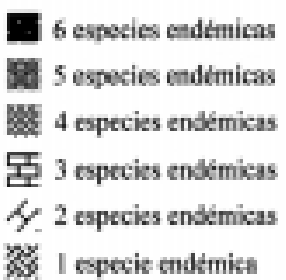

20\% 1 espocie endemica

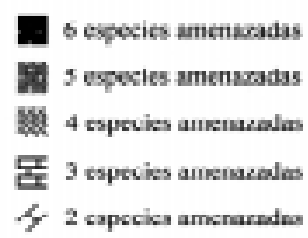

KE lespecie amenazada

Fig. 3: Riqueza de especies endémicas (A) y especies amenazadas (B) en cada cuadrícula. Las cuadriculas en blanco denotan la ausencia de gipsófitos endémicos o amenazados en la cuadrícula.

Endemism (A) and threatened (B) species richness in each cell. Empty cells denote absence of endemic or threatened gypsophytes in the cell. 
4B). Las cuadrículas WF59 y WF79 obtuvieron valores de 0,33 , mientras que el resto de las cuadrículas alcanzaron valores muy inferiores.

\section{Complementariedad: riqueza total de especies}

La cuadrícula que mayor número de especies aportaría a una futura red de reservas de flora gipsícola sería la WG70 (Sorbas), ya que alberga 13 de las 19 especies de gipsófitos del área. La adición de la cuadrícula WG60 (Venta de los Yesos) permitiría la inclusión de otros tres gipsófitos ausentes en la primera. Finalmente, los afloramientos de la WG79 (Topares) recogen las otras tres especies que están ausentes en las primeras dos cuadrículas, con lo que todas las especies de la región quedarían representadas en estas tres localidades (Tabla 2). El resto de combinaciones posibles para lograr la representación de todas las especies exigiría la consideración de un mayor número de localidades.
$\mathbf{A}$

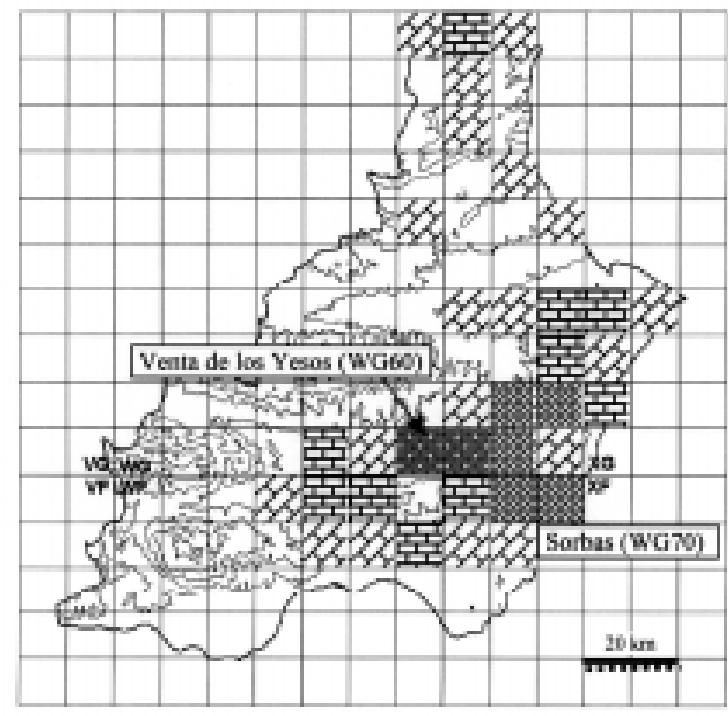

B

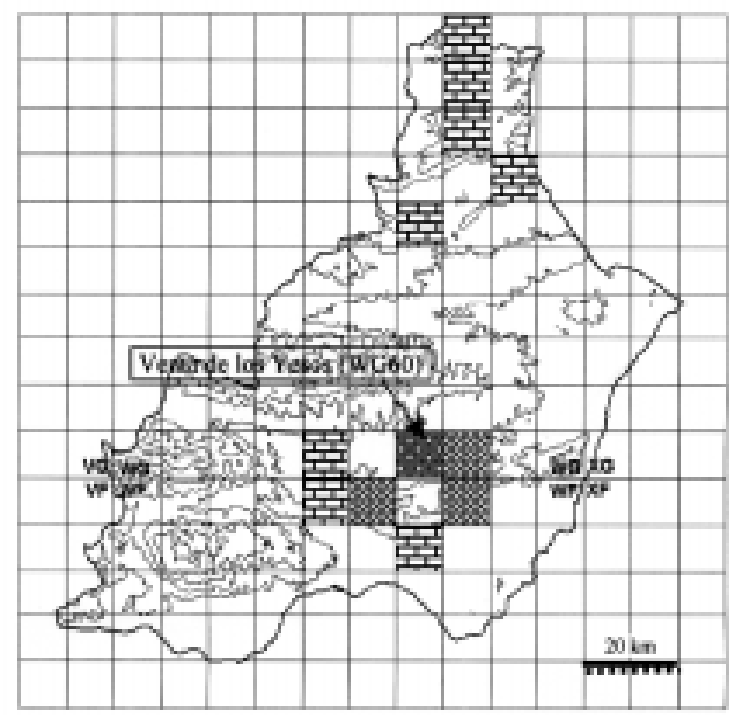

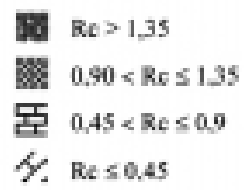

7. Res 50,45

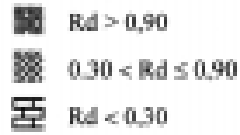

RA $<00.30$

Fig. 4: Valores de rareza continua -Rc- (A) y rareza discontinua-Rd- (B) en cada cuadrícula. Los cuadros en blanco tomaron el valor cero.

Values for continuous -Rc- (A) and discontinuous -Rd- (B) rarity in each cell. Empty cells scored zero. 
Complementariedad: riqueza de endemismos

En este caso, puesto que cada una de las cuadrículas WG70, WG80, WG81, WG91, WF89, XG01 y WF99 contienen a cinco elementos endémicos de la provincia (Chaenorrhinum grandiflorum, Coris hispanica, Helianthemum alypoides, $H$. $x$ mariano-salvatoris y Teucrium turredanum) la selección de cualquiera de ellas permitiría que el $83 \%$ de los endemismos estuviesen representados al menos una vez. La única especie que no quedaría representada en estos enclaves es Rosmarinus x lavandulaceus, que aparece en tres cuadrículas (Tabla 1), de las que Venta de los Yesos (WG60) contiene un mayor número de endemismos (Tabla 2).

\section{Complementariedad: riqueza de especies amena-} zadas

Como se indicó anteriormente, Sorbas (WG70) aportaría seis de los siete taxa amenazados de la región (Tabla 1). La única especie amenazada que quedaría por incluir es Teucrium balthazaris, presente en cuatro localidades: WG79, WG92, WG93 y XG03 (Tabla 2).

Complementariedad: riqueza de especies muy raras

La cuadrícula con mayor acumulación de taxa muy raros fue la WG60, con tres especies (Campanula fastigiata, Frankenia thymifolia y
Rosmarinus x lavadulaceus) de las cinco consideradas como muy raras. La adición de la cuadrícula WG79 del Norte de la provincia (yesos de Topares) permitiría la representación de las otras dos especies más raras, Teucrium balthazaris y Jurinea pinnata (Tabla 2). Una vez incluida la WG60 en la red, aunque la localidad de Sorbas presentó un mayor valor de Rd para las especies muy raras $(\mathrm{Rd}=0,5)$ aportaría sólo una nueva especie frente a las dos especies de Topares.

Selección de áreas prioritarias y declaración de la red de reservas

Los resultados mostraron que a pesar de que la protección de Sorbas se fundamentó principalmente en sus valores geomorfológicos, este enclave también es el de mayor relevancia para la preservación de un buen número de gipsófitos, tanto por su riqueza específica como por su concentración en especies endémicas y amenazadas. Por otro lado, la protección completa de WG80 y WG81 resultaría apropiada si el objetivo de conservación fuese preservar al menos las cuadrículas con mayor riqueza total, con mayor número de especies endémicas o bien con mayor concentración en elementos amenazados. Hay que indicar que en cualquier caso, no todos los afloramientos de estas dos localidades están dentro del área protegida y que las poblaciones de Astragalus grossii quedan fuera del Paraje Natural. No obstante, aunque estos depósitos sólo se incluyeron parcialmente en la reserva, la protección de esos enclaves ha permitido que la mayoría de especies

TABLA 2

Resultados del análisis de complementariedad según los cuatro criterios utilizados

Results of complementarity analysis according to the criteria of conservation used

\begin{tabular}{llcc}
\hline Criterio empleado & Localidades (UTM) & $\begin{array}{c}\text { Número de especies } \\
\text { que aporta la localidad }\end{array}$ & $\begin{array}{c}\text { Número de especies } \\
\text { representadas en la red }\end{array}$ \\
\hline Riqueza total de especies & WG70 (Sorbas) & 13 & 13 \\
& WG60 (Venta de los Yesos) & 3 & 16 \\
& WG79 (Topares) & 3 & 5 \\
Riqueza de endemismos & WG70 (Sorbas) & 5 & 6 \\
& WG60 (Venta de los Yesos) & 1 & 6 \\
Riqueza de especies amenazadas & WG70 (Sorbas) & 6 & 7 \\
& WG79 (Topares) & 1 & 3 \\
Riqueza de especies muy raras & WG60 (Venta de los Yesos) & 3 & 5
\end{tabular}


endémicas y amenazadas de la región queden representadas más de una vez.

Otra serie de cuadrículas empataron con estas tres localidades en cuanto a su número total de especies (WG60), número de endemismos (WG91, WF89, XG01 y WF99) y número de especies amenazadas (WF89). Por ello, la elección entre las celdas de cada uno de estos grupos presenta cierta flexibilidad (Ferrier et al. 2000). En este caso, ya que WG70, WG80 y WG81 lindan entre sí, su elección sí podría resultar apropiada si se busca mantener la conectividad actual de estos sistemas -ya discretos de modo natural-, un argumento frecuentemente considerado de interés en la protección de sistemas distribuidos a modo de parches y como consecuencia, con un fuerte carácter insular (Davidson et al. 1996, Squeo et al. 1998).

Sin embargo, con las medidas de protección actuales no se alcanza el objetivo prioritario fijado en este trabajo. Para ello sería imprescindible la protección de los aljezares de la Venta de los Yesos y de Topares. La elección de Venta de los Yesos permitiría además lograr otra serie de objetivos complementarios. Concretamente permitiría: (1) la preservación de las cuatro cuadrículas más ricas, ya que WG70, WG80 y WG81 ya están protegidas; (2) lograr la preservación de la localidad cuya flora posee el mayor valor de rareza, la representación de dos nuevas especies consideradas como 'muy raras' y el refuerzo de poblaciones de otros gipsófitos, especialmente de Campanula fastigiata, la especie menos frecuente y existente en sólo dos cuadrículas. Actualmente la contribución de los aljezares del Paraje Natural no resulta en absoluto suficiente para lograr estos objetivos; (3) mantener la conectividad natural entre afloramientos de esta zona, ya que la cuadrícula donde se encuadra Venta de los Yesos (WG60) linda con la de Sorbas (WG70) y queda relativamente próxima a la zona protegida.

Finalmente, la inclusión de Topares (WG79) para lograr el objetivo prioritario de representar todas las especies al menos una vez, permitiría a su vez preservar la localidad más diversa de las cuatro que albergan Teucrium balthazaris, elemento endémico del sureste ibérico (exclusivo de las provincias de Almería y Murcia, ambas lindantes entre sí) y que se encuentra catalogado como "en peligro" (Domínguez-Lozano 2000). Hasta ahora, las localidades que concentran poblaciones de este taxon no cuentan con ningún tipo de protección. A la luz de estas consideraciones, en la Fig. 5 se expone la situación de las localidades cuya protección se recomienda y de los enclaves que destacan por los elevados valores en los parámetros de conservación considerados aquí y que, a su vez, se encuentran relativamente próximos entre sí (celdas WF89, WF99, WG91 y XG01).

Es necesario indicar que protegiendo WG92, WG93 o XG03 se protegería también a $T$. balthazaris a la vez que se preservarían poblaciones más próximas al resto de los afloramientos prioritarios. Sin embargo, si se persigue representar todas las especies al menos una vez pero seleccionando los enclaves más próximos al resto de áreas prioritarias, habría que descartar Topares, zona con una riqueza relativamente elevada, y aumentar el número de cuadrículas para incluir a Astragalus grossii (cuyas poblaciones quedan fuera del Paraje Natural) y a Jurinea pinnata. En este caso, conseguir la representación de J. pinnata requeriría siempre la preservación de afloramientos muy alejados del núcleo de localidades seleccionadas hasta ahora. Como mínimo sería necesaria la ampliación del Paraje en las cuadrículas WG80 o WG81 para incluir a Astragalus grossii y la selección de alguna de las cuatro localidades restantes donde aparece J. pinnata, todas ellas muy pobres en gipsófitos (con una o dos especies

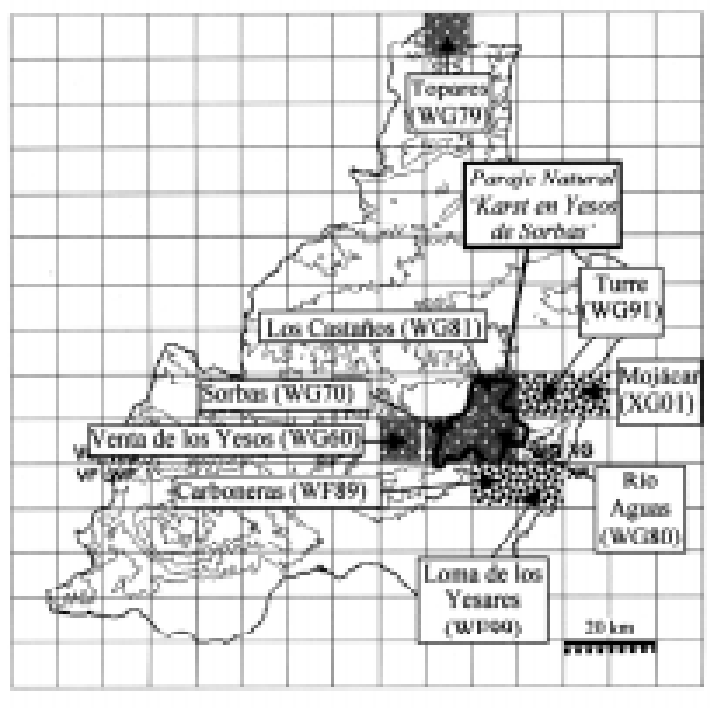

Areas que cumplen los ebjotives priaritanios propuestas para sa protección.

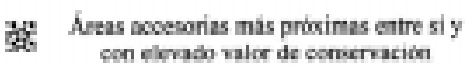

Fig. 5: Localización de las áreas propuestas para la red de conservación. Se muestran también aquellos enclaves con elevado interés para la conservación y los límites del "Paraje Natural del Karst en Yesos de Sorbas".

Location of areas proposed for the conservation-network. Also shown are those locations with high conservation value as well as the limits of the "Paraje Natural del Karst en Yesos de Sorbas". 
como máximo). Teniendo en cuenta las dificultades existentes para proteger un creciente número de enclaves sería posible iniciar una primera fase de ampliación del Paraje Natural en la zona que se indica, sacrificando ligeramente el objetivo de conservación prioritario propuesto, dejando fuera a $J$. pinnata que no es endémica ni está amenazada y que además se comporta como un gipsóvago preferencial, estudiando la posibilidad de incluir esta especie en un futuro y el modo en que debiera efectuarse.

\section{Consideraciones sobre la protección de la flora gipsícola de Almería}

Desde el punto de vista práctico, si se tiene en cuenta la realidad del territorio de estudio, no todas las opciones resultan igualmente viables, aunque desde el análisis puramente numérico existan algunos escenarios más o menos equivalentes. Sin duda el proceso de ampliación del Paraje Natural hasta recoger todos los afloramientos de WG80 y WG81 no estaría exento de dificultades, ya que en la zona de Los Castaños existe una cantera colindante con el límite oriental del espacio natural protegido. La ampliación pasaría probablemente por la permuta de los terrenos con características geológicas semejantes y de menor interés florístico, y, en caso necesario, por la expropiación forzosa de las tierras. Por ello sería necesario estudiar con profundidad los costes económicos y sociales de cada opción. Debido a la oposición de los sectores económicos y sociales implicados y las dificultades legislativas asociadas, parece realmente difícil que la administración andaluza pueda llevar a cabo este tipo de actuaciones a medio plazo. En este caso, el desarrollo de planes de restauración que tengan en cuenta las particularidades de estos ambientes podría contribuir a atenuar los efectos destructivos de la minería en los límites de la reserva (Mota et al. 1999, Dana 2000).

Por el momento, parece más factible la protección de los depósitos de Venta de los Yesos -y quizás también Topares y alguna otra localidad puntual escogida entre las destacadas aquí- para preservar el resto de las especies. En principio en este segundo tipo de situaciones en las que los depósitos prioritarios no se explotan actualmente y que no cuentan con ninguna protección, una figura posiblemente adecuada sería la de "Microreserva de Flora", que resulta especialmente apropiada para la preservación de sistemas naturales discretos distribuidos a modo de parche (Gómez-Campo \& Herranz-Sanz 1993) y que se ha utilizado en otras zonas de España (Laguna
1995, Laguna et al. 1998). Mediante esta figura la Administración Autonómica cuya legislación contemple esta figura, compra pequeños espacios de gran valor biológico -o bien compensa económicamente al propietario por su protección- gestionando su conservación. La reducida extensión de las áreas seleccionadas (unos 3,7 km² en Venta de los Yesos) y el hecho de enclavarse en zonas con bajas densidades de población, haría muy factible la adquisición de una buena parte de los terrenos por la administración, de modo que ésta gestione y garantice su conservación a largo plazo.

Sin embargo, por el momento, la legislación ambiental de la Comunidad Autónoma Andaluza no contempla la existencia de este tipo de espacios, por lo que a las dificultades prácticas a las que se enfrenta la conservación de tan valiosos enclaves, se le une la ausencia de figuras de protección específicamente creadas para este tipo de sistemas discontinuos y de reducida extensión. Por ello es urgente una ampliación de las figuras de protección previstas por la legislación ambiental andaluza que tenga en cuenta los distintos tipos de ecosistemas existentes en el sur de España y que permita incluir la figura de Microreserva.

\section{AGRADECIMIENTOS}

Agradecemos profundamente los valiosos comentarios realizados y las ideas aportadas por dos revisores anónimos que mejoraron notablemente el manuscrito inicial. Este trabajo fue financiado por la DGICYT -PB95-0959- y por la concesión de una beca de investigación financiada por la empresa HISALBA (Hornos Ibéricos Alba, SA) a uno de los autores.

\section{LITERATURA CITADA}

ANON (1992) Directive 92/43/CEE of the Council of the European Community on the Conservation of Habitats and Wild Fauna and Flora. European Community, Brussels, Belgium DOCE 206/1992, serie L: 7-50.

BRAUN-BLANQUET J \& O BOLÒS (1957) Les groupements végétaux du bassin moyen de l'Ebre et leur dynamisme. Anales de la Estación Experimental del Aula Dei 5: 1-266.

CABELLO J (1997) Factores ambientales, estructura y diversidad en comunidades de matorral de ambiente semiárido (Tabernas-Sierra Alhamilla-Níjar, SE Ibérico). Tesis Doctoral, Facultad de Ciencias Experimentales, Universidad de Almería, Almería, España. $612 \mathrm{pp}$.

CALAFORRA JM (1998) Karstología de yesos. Monografías Ciencia y Tecnología 3. Universidad de Almería \& Instituto de Estudios Almerienses, Almería, España. 384 pp. 
CALAFORRA JM \& P FORTI (1991) Le Pale di gesso e le stalagmite cave: due nuove forme di concrezionamento gessoso scoperte nelle grotte di Sorbas (Andalusia, Spagna). Le grotte d'Italia 4, 15: 73-88.

CALAFORRA JM \& A PULIDO (1997) Peculiar landforms in gypsum karst of Sorbas (southeastern Spain). Carbonates and Evaporites 12: 110-116.

CASTRO PARGA I, JC MORENO SAIZ, CJ HUMPHRIES \& PH WILLIAMS (1996) Strengthening the National and Natural Park system of Iberia to conserve vascular plants. Botanical Journal of the Linnean Society 121: 189-206.

CERRILLO MI, E DANA, F PÉREZ-GARCÍA, ML RODRÍGUEZ-TAMAYO, AJ SOLA \& JF MOTA (2001b) Consideraciones sobre la conservación de los aljezares almerienses de acuerdo con su riqueza en gipsófitos. En: Cano E, A García, JA Torres \& C Salazar (eds) Valoración y gestión de espacios naturales: 367-376. Universidad de Jaén, Jaén, España.

CERRILLO MI, JF MOTA, FJ PÉREZ-GARCÍA, H CASTRO \& E DANA (2001a) Datos para la conservación de Helianthemum alypoides Losa \& Rivas-Goday. En: Cano E, A García, JA Torres \& C Salazar (Eds) Valoración y gestión de espacios naturales: 105-112. Universidad de Jaén, Jaén, España.

COLWELL RK \& JA CODDINGTON (1994) Estimating terrestrial biodiversity trough extrapolation. Philosophical Transactions of the Royal Society of London Series B 345: 101-118.

CUETO M (1989) Los recursos vegetales de las Sierras de Maria y Orce como base para la gestión de un espacio natural. Tesis Doctoral, Facultad de Ciencias, Universidad de Granada, Granada, España. 495 pp.

DANA E (2000) Estudio de la sucesión vegetal en canteras de yesos abandonadas y su aplicación a la conservación de la flora y vegetación de los aljezares ibéricos. Tesis Doctoral, Facultad de Ciencias Experimentales, Universidad de Almería, Almería, España. 274 pp.

DAVIDSON DW, WD NEWMARK, JW SITES, DK SHIOZAWA, EA RICKART, KT HARPER \& RB KEITER (1996) Selecting wilderness areas to conserve Utah's biological diversity. Great Basin Naturalist 56: 95-118.

DINERSTEIN E \& E WIKRAMANAYAKE (1993) Beyond "hot spots": how to prioritize investments in biodiversity in the Indo-Pacific region. Conservation Biology 7: 53-65

DOMÍNGUEZ-LOZANO F (ed) (2000) Lista Roja de la flora vascular española (Red List of Spanish Vascular Flora). Conservación Vegetal No. 6. Universidad Autónoma de Madrid, Madrid, España. 39 pp.

EGEA JM \& FL ALONSO (1997) Patrones de distribución en la flora liquénica xerófila del sureste de España. Acta Botanica Malacitana 21: 35-47.

ESCUDERO A, LF CARNES \& F PÉREZ-GARCÍA (1997) Seed germination of gypsophytes and gypsovags in semi-arid central Spain. Journal of Arid Environments 36: 487-497.

FERRIER S, RL PRESSEY \& TW BARRETT (2000) A new predictor of the irreplaceability of areas for achieving a conservation goal, its application to realworld planning and a research agenda for further refinement. Biological Conservation 93: 303-325.
GASTON KJ (1997) Rarity. Chapman \& Hall, London, Inglaterra. $205 \mathrm{pp}$.

GÓMEZ-CAMPO C \& J HERRANZ-SANZ (1993) Conservation of Iberian endemic plants: the botanical reserve of la Encantada (Villarrobledo, Albacete, Spain). Biological Conservation 64: 155-160.

GÓMEZ-CAMPO C \& J MALATO-BELIZ (1985) The Iberian Peninsula. In: Gómez-Campo C (ed) Plant conservation in the Mediterranean area: 47-70. Dr. W. Junk, Dordrecht, Holanda.

GUERRA J, RM ROS, MJ CANO \& M CASARES (1995) Gypsiferous outcrops in SE Spain, refuges of rare, vulnerable and endangered Bryophytes and Lichens. Cryptogamie, Bryologie \& Lichénologie 16: 125135.

GUTIÉRREZ L (1990) Estudio de la flora y vegetación liquénica de las zonas yesíferas de la provincia de Almería. Memoria de Licenciatura, Facultad de Ciencias, Universidad de Granada, Granada, España. 209 pp.

GUTIÉRREZ L \& M CASARES (1994) Flora liquénica de los yesos miocénicos de la provincia de Almería (España). Candollea 49: 343-358.

HAILA Y \& KOUKI J (1994) The phenomenon of biodiversity in conservation biology. Annales Zoologi Fennici 31: 5-18.

HERNÁNDEZ HM \& RT BÁRCENAS (1996) Endangered cacti in the Chihuahuan desert: II. Biogeography and conservation. Conservation Biology 10: 1200-1209.

HOWARD PC (1991) Nature conservation in Uganda's tropical forest reserves. World Conservation Union, Gland, Switzerland. 313 pp.

HUMPHRIES CJ, PH WILLIAMS \& RI VANE-WRIGHT (1995) Measuring biodiversity value for conservation. Annual Review of Ecology and Systematics 26: $93-$ 111.

KERR JT (1997) Species richness, endemism, and the choice of areas for conservation. Conservation Biology 11: 1094-1100.

KIRKPATRICK JB (1983) An iterative method for establishing priorities for the selection of nature reserves: an example from Tasmania. Biological Conservation 25: 127-134.

LAGUNA E (1995) Microreservas de flora: un nuevo modelo de conservación en la comunidad valenciana. Quercus, diciembre: 22-26.

LAGUNA E, M CRESPO, G MATEO, S LÓPEZ, C FABREGAT, L SERRA, J HERRERO-BORGOÑÓN, J CARRETERO, A AGUILELLA, I PALESÍ \& R FIGUEROLA (1998) Flora endémica, rara o amenazada de la comunidad valenciana. Generalitat Valenciana. Conselleria de Medioambiente, Valencia, España. $443 \mathrm{pp}$.

LÁZARO R (1984) Contribución al estudio de la flora y vegetación gipsícola de la provincia de Almería. Tesis de Licenciatura, Universidad de Málaga, Málaga, España. 302 pp.

LÁZARO R (1986) Sobre la flora y vegetación gipsícola almeriense (fanerógamas). Boletín del Instituto de Estudios Almerienses 6: 131-150.

LOIDI J \& M COSTA (1997) Sintaxonomía de los matorrales gipsícolas españoles. Fitosociología 32: 221227. 
MARGULES CR, AO NICHOLLS \& RL PRESSEY (1988) Selecting Networks of reserves to maximize biological diversity. Biological Conservation 43: 63-76.

MARGULES CR \& MB USHER (1981) Criteria used in assessing wildlife conservation potential: a review. Biological Conservation 21: 79-109.

MARGULES CR \& MP AUSTIN (1991) Nature conservation: cost effective biological surveys and data analysis. Commonwealth Scientific and Industrial Research Organization, Melbourne, Australia. $207 \mathrm{pp}$.

MOLINA A, A RUBIO-SÁNCHEZ \& A ESCUDERO (1989) Cartografía corológica ibérica. Aportaciones 1 a 4. Botanica Complutensis 15: 243-260.

MONTSERRAT P \& L VILLAR (1972) El endemismo ibérico, aspectos ecológicos y fitotopográficos. Bulletin Society Broteriana, Serie 2, 46: 503-527.

MORENO-SAIZ JC, I CASTRO-PARGA, CJ HUMPHRIES \& PH WILLIAMS (1996) Strengthening the National and Natural Park system of Iberia to conserve pteridophytes. En: Camus JM, M Gibby \& RJ Johns (eds) Pteridology in perspective: 101-123. Royal Botanic Gardens, Kew, Inglaterra.

MORENO-SAIZ JC, I CASTRO-PARGA \& H SAINZOLLERO (1998) Numerical analyses of distributions of Iberian and Balearic endemic monocotyledons. Journal of Biogeography 25: 179-194.

MOTA JF, JJ ALVARADO, F GÓMEZ, F VALLE \& J CABELLO (1993) Vegetación gipsícola y conservación de la naturaleza. Colloques Phytosociologiques 21: 677-688.

MOTA JF, J CABELLO, M CUETO, F GÓMEZ, E GIMÉNEZ \& J PEÑAS (1997) Datos sobre la vegetación del sureste de Almería (Desierto de Tabernas, Karst en yesos de Sorbas y Cabo de Gata). Universidad de Almería, Almería, España. 130 pp.

MOTA JF, ML RODRÍGUEZ-TAMAYO, J PEÑAS, FJ PÉREZ-GARCÍA, E DANA \& ME MERLO (1998) Examen de la vegetación de los aljezares ibéricos con especial atención a la provincia de Almería. Investigación \& Gestión 3: 147-158.

MOTA JF, E MERLO, C OYONARTE, J PEÑAS, J PÉREZGARCÍA, ML RODRÍGUEZ-TAMAYO, A AGUILERA, G BONILLA, J CABELLO, M CUETO, E DANA, MA FERNÁNDEZ-JURADO, A SOLA \& MI CERRILLO (1999) Vegetación, sucesión y restauración ecológica en canteras de yeso. En: Navarro A, JA Sánchez-Garrido \& DM Collado (eds) Minería y medio ambiente en la cuenca mediterránea: 83-93. Servicio de Publicaciones de la Universidad de Almería, Almería, España.

MYERS N (1988) Threatened biotas: "hotspots" in tropical forest. The Enviromentalist 8: 187-208.

MYERS N (1990) The biodiversity challenge: expanded hot-spots analysis. The Environmentalist 10: 243256.

MYERS N, RA MITTERMEIER, CG MITTERMEIER, GA FONSECA \& J KENT (2000) Biodiversity hotspots for conservation priorities. Nature 403: 853858.

NIETO FELINER G (1999) Vascular plant distribution in the Iberian Peninsula and the Balearic Island: current projects. Acta Botanica Fennica 162: 23-33.
OSTERMANN OP (1998) The need for management of nature conservation sites designated under Natura 2000. Journal of Applied Ecology 35: 968-973.

PRENDERGAST JR, RM QUINN, JH LAWTON, BC EVERSHAM \& DW GIBBONS (1993) Rare species, the coincidence of diversity hotspots and conservation strategies. Nature 365: 335-337.

PRESSEY RL, CJ HUMPHRIES, CR MARGULES, RI VANE-WRIGHT \& PH WILLIAMS (1993) Beyond opportunism: key principles for systematic reserve selection. Trends in Ecology and Evolution 8: 124128.

RIVAS-GODAY S (1957) Aportaciones a la fitosociología hispánica. Nota II. Comunidades gipsófilas del centro y sudeste de España. Anales del Instituto de Botánica Cavanilles (España) 14: 433-500.

RIVAS-MARTÍNEZ S \& M COSTA (1970) Comunidades gipsícolas del centro de España. Anales del Instituto Botánico Cavanilles (España) 27: 193-224.

RUBIO-SÁNCHEZ A (1990) Cartografía corológica ibérica. Aportación 24. Botanica Complutensis (España) 16: $156-159$.

RUBIO-SÁNCHEZ A \& A ESCUDERO (1992) Cartografía corológica ibérica. Aportación 35. Botanica Complutensis (España) 17: 180-187.

RUBIO-SÁNCHEZ A, A ESCUDERO \& A MOLINA (1992) Cartografía corológica ibérica. Aportación 36 y 39. Botanica Complutensis (España) 17: 188-201.

SAGREDO R (1987) Flora de Almería. Plantas vasculares de la provincia. Instituto de Estudios Almerienses. Diputación de Almería, Almería, España. 555 pp.

SANZ F \& M COSTA (1987) Catálogo florístico del sector septentrional de la Sierra del Cabo de Gata. Boletín del Instituto de Estudios Almerienses 7: 259-270.

SIMÓN JC (1994) La flora vascular española: diversidad y conservación. Ecología 8: 203-225.

SQUEO FA, LA CAVIERES, G ARANCIO, JE NOVOA, O MATTHEI, C MARTICORENA, R RODRÍGUEZ, MTK ARROYO \& M MUÑOZ (1998) Biodiversidad de la flora vascular en la región de Antofagasta, Chile. Revista Chilena de Historia Natural 71: 517591.

USHER MB (1986) Wildlife conservation evaluation. Chapman \& Hall, Londres, Inglaterra. 394 pp.

VANE-WRIGHT RI, CJ HUMPHRIES \& PH WILLIAMS (1991) What to protect?-Systematics and the agony of choice. Biological Conservation 55: 235-254.

WILLIAMS PH (1993) Measuring more of biodiversity for choosing conservation areas, using taxonomic relatedness. En: Moon TY (ed) International Symposium on Biodiversity and Conservation: 194227. Korea University, Seoul, Korea del Sur.

WILLIAMS PH (1994) Biodiversity value and taxonomic relatedness. En: Hochberg ME, J Clobert \& R Babault (eds) Aspects of the genesis and maintenance of biological diversity: 261-277. Oxford University Press, Oxford, Inglaterra.

WILLIAMS PH (1999) Key sites for conservation: areaselection methods for biodiversity. En: Mace GM, A Balmford \& JR Ginsberg (eds) Conservation in a changing world: integrating processes into priorities for action: 211-249. Cambridge University Press, Cambridge, Inglaterra. 
WILLIAMS PH, D GIBBONS, C MARGULES, A REBELO, C HUMPHRIES \& R PRESSEY (1996a) A comparison of richness hotspots, rarity hotspots, and complementary areas for conserving diversity of british birds. Conservation Biology 10: 155-174.
WILLIAMS PH, GT PRANCE, CJ HUMPHRIES \& KS EDWARDS (1996b) Promise and problems in applying quantitative complementary areas methods for representing the diversity of some Neotropical plants (families Dichapetalaceae, Lecythidaceae, Caryocaraceae, Chrysobalanaceae and Proteaceae). Biological Journal of the Linnean Society 58: 125157.

Editor Asociado: P. Marquet

Recibido el 8 de noviembre de 2000; aceptado el 7 de febrero de 2002 